\title{
Marginally turbulent flow in a square duct
}

\author{
By MARKUS UHLMANN ${ }^{1}$, ALFREDO PINELLI, \\ GENTA KAWAHARA AND ATSUSHI SEKIMOTO ${ }^{2}$ \\ ${ }^{1}$ Modeling and Numerical Simulation Unit, CIEMAT, 28040 Madrid, Spain \\ ${ }^{2}$ Department of Mechanical Science, Osaka University, 560-8531 Osaka, Japan
}

(Received 29 June 2007)

Direct numerical simulation of turbulent flow in a straight square duct was performed in order to determine the minimal requirements for self-sustaining turbulence. It was found that turbulence can be maintained for values of the bulk Reynolds number above approximately 1100, corresponding to a friction-velocity-based Reynolds number of 80 . The minimum value for the streamwise period of the computational domain measures around 190 wall units, roughly independently of the Reynolds number. Furthermore, we present a characterization of the flow state at marginal Reynolds numbers which substantially differs from the fully turbulent one. In particular, the marginal state exhibits a 4-vortex secondary flow structure alternating in time whereas the fully turbulent one presents the usual 8-vortex pattern. It is shown that in the regime of marginal Reynolds numbers buffer layer coherent structures play a crucial role in the appearance of secondary flow of Prandtl's second kind.

\section{Introduction}

Turbulent flow in a plane channel has been extensively studied in the past and many aspects of its dynamics are by now fairly well understood, at least as far as the near-wall region is concerned. The flow in a duct with a rectangular cross-section, on the other hand, has received much less attention. Although its geometry is only mildly more complex, it exhibits important additional phenomena, of which the most prominent one is the appearance of turbulence-driven secondary motion in the cross-sectional plane. In particular, in this paper we will address the canonical case of ducts with square section. The appearance of secondary mean motion of a turbulent flow in such a geometry is well known since the experiments by Nikuradse (1926) who was the first to measure it indirectly. One significant consequence of the secondary mean motion is a non negligible deformation of the primary mean velocity profile. Previous experimental measurements of the flow in a square duct (Brundrett \& Baines 1964; Gessner 1973; Melling \& Whitelaw 1976) as well as direct numerical simulations (Gavrilakis 1992; Huser \& Biringen 1993) have provided useful reference data for the mean velocities and the Reynolds stress tensor. However, those studies were mainly focused upon the budget of the averaged flow equations, while not providing much information on the underlying physical mechanisms responsible for the formation of secondary flow. Although some insight about vortex kinematics in duct flow was provided by Kawahara \& Kamada (2000), a detailed investigation of the dynamics of coherent structures in such flow has to our knowledge not been reported in the literature.

Galletti \& Bottaro (2004) and Bottaro, Soueid \& Galletti (2006) have employed a parabolized linear formulation (in the latter reference a simple mixing-length model accounts for the Reynolds stresses) to describe the transient growth of a perturbation field 
which under certain circumstances resembles the experimentally observed secondary flow pattern in the square duct. However, their linear analysis of the mean field seems to be more appropriate to model a transitional path toward turbulence rather than to describe a fully developed turbulent state.

In the present study we numerically investigate marginal turbulence states in a square duct, meaning that we focus upon flows developing at low Reynolds numbers just above the minimum value for sustained turbulence. The rationale behind this choice is that in this regime the cross-stream scale of coherent structures should be comparable with the duct width. Therefore, such a scenario should be an ideal one to elucidate possible direct relationships between well known buffer layer coherent structures (i.e. streaks and streamwise vortices) and cross stream mean motion.

The first objective of the research has been the quantitative determination of marginal states. Subsequently, we have performed a detailed study to characterize these states. It is found that the marginal state is substantially different from the fully turbulent one displaying periods of strong turbulent activity and quasi laminar behavior switching from a pair of facing walls to the other. Finally, we have addressed the generation mechanism of turbulent mean secondary motion from the point of view of coherent structure dynamics at marginal Reynolds numbers.

The organization of this paper is as follows. In $\S 2$ a pseudo-spectral direct numerical simulation method by which flow data are generated is presented. We then proceed, in $\S 3$, with an identification of the critical parameter values above which turbulence can be sustained in the square duct. In $\S 4$ we present the characterization of the flow under marginal conditions in terms of statistics and newly discovered secondary flow patterns with reduced symmetries. We also describe statistical results stemming from a coherent vortex eduction study. Finally, in $§ 5$, a general discussion about the obtained results is presented together with some conclusions.

\section{Numerical methodology}

We are considering the flow in a straight duct with square cross-section of half-width $h$. The Cartesian coordinates are $x, y, z$ with the origin located in the center of the duct and $x$ defined along the streamwise direction. The flow field is assumed to be streamwise periodic over a period of length $L_{x}$ and a constant flow rate is imposed at each time step (Pinelli et al. 2007).

We solve the incompressible Navier-Stokes equations with the velocity components $u, v, w$ (along $x, y$ and $z$ directions, respectively) and the pressure $p$ (normalized by a unit density) as the independent variables. An incremental-pressure projection method is used for solving the momentum equation and imposing the divergence-free condition in three fractional steps. The temporal integration is based upon the Crank-Nicholson scheme for the viscous terms and a three-step low-storage Runge-Kutta method for the non-linear terms, including the pressure gradient. This time discretization is identical to the one described by Verzicco \& Orlandi (1996) with a time accuracy of $\mathcal{O}\left(\Delta t^{2}\right)$ in the interior of the domain. On the walls the impermeability constraint is exactly enforced, while -due to the use of the fractional step scheme- the no-slip condition is verified up to an error of $\mathcal{O}\left(\Delta t^{2} \nu\right)$, where $\nu$ is the kinematic viscosity. In all our simulations this "slip error" was kept below $10^{-4}$ times the bulk flow velocity by adjusting the time step $\Delta t$ accordingly.

The flow variables are expanded in terms of truncated Fourier series in the streamwise direction and Chebyshev polynomials in the two cross-stream directions. We use a collocated grid arrangement in physical space, constructed from an equidistant spacing in the 
$x$-direction and the Chebyshev-Gauss-Lobatto points in $y, z$. The non-linear terms in the momentum equation are evaluated in physical space whereas the explicit contribution of the linear terms is evaluated in spectral space. The fields are transformed back and forth by means of fast Fourier transform and fast cosine transform. De-aliasing according to the $2 / 3$-rule is performed in the streamwise direction. For each streamwise Fourier mode we need to solve four two-dimensional Helmholtz equations at every Runge-Kutta substep; the corresponding solution is carried out by a fast diagonalization technique applied to the discrete Laplace operator (Haldenwang et al. 1984). This approach yields four spurious pressure modes (Leriche \& Labrosse 2000), which, however, do not affect the velocity field since their first derivatives in all three coordinate directions vanish identically at all interior collocation nodes. For the purpose of post-processing the pressure field, these modes can simply be filtered in Chebyshev space.

We impose a time-independent volume flow rate $Q$ which is related to the streamwise velocity and the bulk velocity $u_{b}$ by: $Q=\int_{-h}^{h} \int_{-h}^{h} u \mathrm{~d} y \mathrm{~d} z=u_{b} 4 h^{2}$. As a consequence, the Reynolds number based upon the bulk velocity and duct half-width, $R e_{b}=u_{b} h / \nu$, is fixed a priori, while the wall-shear stress fluctuates in time. The instantaneous wall-shear stress at each cross-section is defined as follows:

$$
\tau_{w}(x, t)=-\frac{\nu}{8 h}\left(\int_{-h}^{h}\left[\frac{\partial u}{\partial y}\right]_{y=-h}^{y=h} \mathrm{~d} z+\int_{-h}^{h}\left[\frac{\partial u}{\partial z}\right]_{z=-h}^{z=h} \mathrm{~d} y\right) .
$$

For later convenience, let us define a number of averaging operators. The time average is denoted by an overbar, e.g. $\bar{\tau}_{w}=\frac{1}{t_{2}-t_{1}} \int_{t_{1}}^{t_{2}} \tau_{w} \mathrm{~d} t$, the streamwise average is defined as $\langle u\rangle_{x}(y, z, t)=\frac{1}{L_{x}} \int_{0}^{L_{x}} u \mathrm{~d} x$, the average over the cross-section is defined as $\langle u\rangle_{y z}(x, t)=$ $\frac{1}{4 h^{2}} \int_{-h}^{h} \int_{-h}^{h} u \mathrm{~d} y \mathrm{~d} z$, and the average over the streamwise direction and time as $\langle u\rangle(y, z)=$ $\overline{\langle u\rangle}_{x}$. Fluctuations are defined with respect to the streamwise-and-time average, $u=$ $\langle u\rangle+u^{\prime}$, and with respect to the streamwise average only, $u=\langle u\rangle_{x}+u^{\prime \prime}$. Finally we introduce the mean friction velocity $u_{\tau}=\sqrt{\left\langle\tau_{w}\right\rangle}$ which is used to define the usual viscous wall units indicated in the following by the superscript + .

For all simulations presented below we have enforced the following resolution requirements: the time step was chosen such that the CFL number is below 0.3, the number of Fourier modes such that the streamwise grid spacing $\Delta x^{+}$is below 15 , the number of Chebyshev modes such that the maximum grid size in the cross-stream directions $\Delta y^{+}=\Delta z^{+}$is below 5.7. In most cases, especially the marginal ones which are of particular interest here, the spatial and temporal resolution was in fact considerably higher.

Finally, it should be mentioned that all the statistical data, presented in the paper, have been obtained considering at least an accumulation time of $1500 \mathrm{~h} / \mathrm{u}_{\mathrm{b}}$.

A complete description of the numerical technique and an exhaustive validation will be given in Pinelli et al. (2007).

\section{Identification of the critical conditions}

The purpose of the minimization study is to determine the critical parameter values which allow for sustained turbulence. Here the two significant quantities are the Reynolds number and the length of the streamwise period. We have performed simulations for various values of both quantities, covering a total of 70 parameter points in the range $0.79 \leq L_{x} / h \leq 25.13$ and $320 \leq R e_{b} \leq 2600$. The results from this series provide quantitative information about the scaling properties of the coherent structures involved in the regeneration mechanism of near-wall turbulence in the duct geometry. 
$(a)$

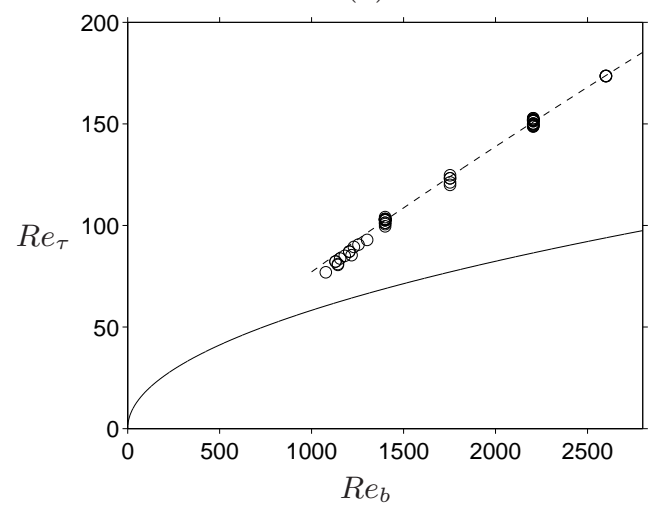

$(b)$

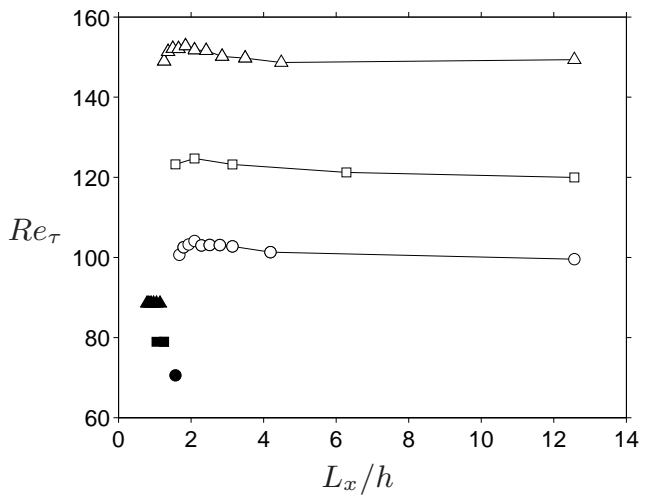

FIGURE 1. (a) Variation of the wall friction as a function of the bulk Reynolds number: $\circ$, present results (turbulent); - - - empirical formula of Jones (1976); —- laminar flow. (b) The variation of the wall friction as a function of the streamwise period for three different Reynolds numbers: $\circ, R e_{b}=1400 ; \square, R e_{b}=1753 ; \Delta, R e_{b}=2205$. Filled symbols are for laminar flow.

Each simulation was initiated with a fully developed turbulent flow field taken from a previous run. Care was taken to vary as little as possible the values of the parameters between successive simulations (Jiménez \& Moin 1991). The computations were run until either the flow relaminarized or a minimum of 2000 bulk flow time units had elapsed, while the sustenance of turbulence was verified by monitoring the temporal variation of the box-averaged kinetic energy fluctuations. This lower limit for the integration time is comparable to the values used for the determination of the critical conditions in plane channel flow by Jiménez \& Moin (1991) and in pipe flow by Faisst \& Eckhardt (2004).

Figure 1(a) shows the variation of the wall friction in terms of the friction-velocitybased Reynolds number, $R e_{\tau}=u_{\tau} h / \nu$, as a function of the bulk Reynolds number $R e_{b}$. The curve for the laminar state is given by $R e_{\tau}=\sqrt{a R e_{b}}$ with $a=3.3935$ (Tatsumi \& Yoshimura 1990). It can be observed that a turbulent state can be maintained only above $R e_{b}=1077$. This value should be compared to the corresponding value of 1000 for the plane channel configuration (Carlson, Widnall \& Peeters 1982) and 1125 for pipe flow (Faisst \& Eckhardt 2004).

At this critical point we obtain $R e_{\tau}=77$ which means that the width of the duct in turbulent conditions corresponds to at least 154 viscous units. In other words, the critical Reynolds number sets a lower limit for the lateral extension of the duct in wall scaling. This is an important difference with respect to the plane geometry, where the spanwise size of the computational domain can be freely adjusted and, therefore, the minimization experiment can be used to determine the natural scaling of near-wall structures in the spanwise direction (Jiménez \& Moin 1991; Hamilton, Kim \& Waleffe 1995). Conversely, the presence of a pair of side walls in the square duct always imposes a spanwise constraint upon the flow developing near a given wall plane. The implications of these geometrical constraints upon the dynamics of the coherent structures will be discussed in more detail in $\S 5$. Figure 1 $(a)$ also shows that the friction velocity in the turbulent regime grows almost linearly with the bulk velocity. Our data is very well represented by the empirical correlation of Jones (1976), $f^{-\frac{1}{2}}=2 \log _{10}\left(2.25 R e_{b} f^{\frac{1}{2}}\right)-0.8$, where the friction factor $f$ is defined as $f=8 u_{\tau}^{2} / u_{b}^{2}$. Note that in the turbulent regime slightly different values for $R e_{\tau}$ are obtained at a given value of $R e_{b}$ when the streamwise length $L_{x}$ is varied. This dependence of the wall friction upon the length of the streamwise period is shown in figure $1(b)$ for three different values of the bulk flow Reynolds number. In all cases, 


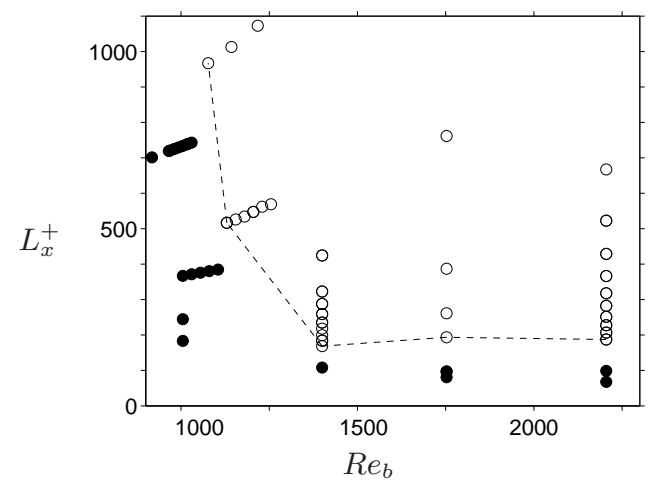

Figure 2. Map of turbulent (o) and laminar $(\bullet)$ flow states in the plane defined by the length of the streamwise period of the computational box (measured in wall units) and the bulk Reynolds number. The dashed lines indicate the border of the region where turbulence can be sustained.

$R e_{\tau}$ exhibits a mild peak just above the critical value for $L_{x}$ and then tends towards an asymptotic value for larger domains. We will return to this point at the end of the section.

The map of turbulent/laminar flow states in the plane spanned by $R e_{b}$ and $L_{x}^{+}$is given in figure 2. It can be observed that the critical value for the streamwise period is roughly independent of the Reynolds number when expressed in wall units. In particular, the shortest streamwise period with sustained turbulence for the three values $R_{e}=$ $1400,1753,2205$ corresponds to values just below 200 wall units $\left(L_{x}^{+}=168,193,187\right.$ respectively). As a comparison, the corresponding length in outer scales varies by a factor 1.33 over the same range $\left(L_{x} / h=1.68,1.57,1.26\right)$, which suggests that wall scaling is more adequate for the minimal periodic cell. For the plane channel configuration, Jiménez \& Moin (1991) have observed a similar although slightly higher minimum length of 250350 wall units. However, for a consistent comparison between the two geometries one should consider that mean skin friction is not uniform along the perimeter in the case of the duct (cf. Gavrilakis 1992, figure 7). Using a different definition of the global skin friction (e.g. the value at the wall bi-sector) would lead to higher estimates of critical $L_{x}^{+}$, closer to the value of Jiménez \& Moin (1991).

For computational boxes moderately larger than the critical value, at all time quasi streamwise vortices span most of the domain, thus increasing the skin friction value. Conversely, for even longer boxes the population of vortices per unit length diminishes, thus decreasing the integral value of skin friction. This reasoning explains the local maxima of skin friction as observed in figure 1(b).

\section{Characterisation of the marginal state}

\subsection{Secondary flow patterns}

The commonly observed pattern of mean secondary flow in the cross-plane of the square duct consists of eight vortices, one counter-rotating pair being located above each of the four wall planes. Their sense of rotation is such that the secondary flow on the diagonals is directed towards the corners (cf. Gavrilakis 1992).

In the course of the present investigation it was observed that the flow under marginal conditions (i.e. at Reynolds numbers close to the critical values given in figure 2) can exhibit a pattern with only four dominant mean secondary vortices over substantial intervals of time, as shown in figure 3 . The 4 -vortex state is characterized by two pairs 
(a)

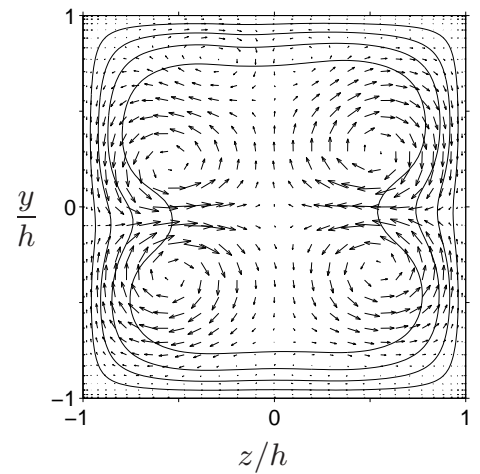

(b)

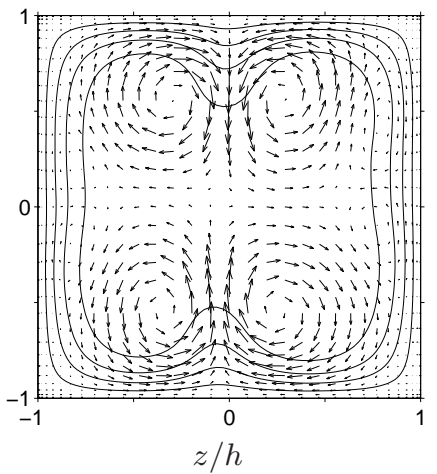

(c)

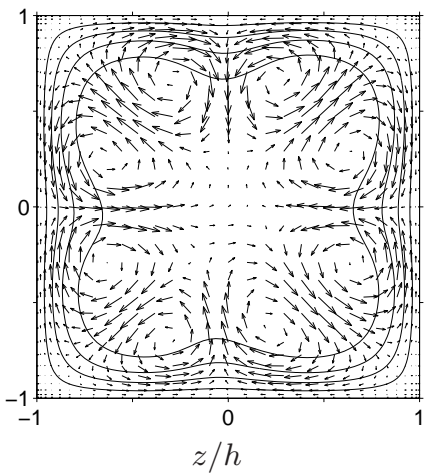

Figure 3. Contour lines of the primary mean flow $\langle u\rangle$ (with increment $\max \langle u\rangle / 5$ ) and vectors of the secondary mean flow $\langle v\rangle,\langle w\rangle$ for $R e_{b}=1205$ and $L_{x} / h=2 \pi$ : $(a)$ averaging interval $771 h / u_{b} ;(b)$ a different interval with length $482 h / u_{b} ;(c)$ long-time integration including both former intervals $\left(1639 h / u_{b}\right)$. Vectors are shown for every third grid point.

of counter-rotating vortices associated to a pair of opposite walls. The sense of rotation of the individual vortices is consistent with the usual 8-vortex pattern. For symmetry reasons, 4 -vortex patterns with two different orientations exist, corresponding to secondary flow vortices located near the planes $z / h= \pm 1$ and $y / h= \pm 1$, respectively (cf. figure $3 a, b)$. We have observed that the flow state indeed switches from one orientation to the other during the temporal evolution, leading to a long-time average mean flow exhibiting the common 8-vortex pattern as shown in figure $3(c)$.

Flow visualizations of instantanous flow fields exhibiting the 4 -vortex state show that turbulence activity is concentrated mostly on one pair of opposite walls, while the flow near the other two walls is much more quiescent. During those intervals, a single lowvelocity streak is located around the bisector of each one of the 'active walls', whereas typically only very weak structures are detected on the other pair of parallel walls. Near the active walls, each streak is flanked by staggered streamwise vortices of the corresponding signs, as often observed in plane channel flows (see Jeong et al. 1997). Therefore, the mean flow near the active wall exhibits a pair of counter-rotating streamwise vortices when the flow field is averaged over intervals of $\mathcal{O}(100)$ bulk flow time units. We shall further discuss the relation of streaks and vortices with the mean flow in $\S 4.2$.

In order to quantitatively identify the 4 -vortex state, we compute the integral of the square of the streamwise-averaged streamwise component of vorticity in the crosssectional plane, the integration extending over one of the four triangular regions delimited by the diagonals, viz:

$$
S_{i}(t)=\iint_{\Omega_{i}}\left\langle\omega_{x}\right\rangle_{x}^{2} \mathrm{~d} y \mathrm{~d} z
$$

where the triangular regions $\Omega_{i}$ are defined as:

$$
\begin{array}{ll}
\Omega_{1}:\{(y, z) \mid y<z \cap y<-z\}, & \Omega_{3}:\{(y, z) \mid y>z \cap y>-z\}, \\
\Omega_{2}:\{(y, z) \mid y<z \cap y>-z\}, & \Omega_{4}:\{(y, z) \mid y>z \cap y<-z\} .
\end{array}
$$

When considering non-laminar states, we introduce the following dimensionless indicator function:

$$
I(t) \equiv \frac{S_{1}+S_{3}-S_{2}-S_{4}}{S_{1}+S_{2}+S_{3}+S_{4}}
$$

bounded by $-1 \leq I \leq 1$. When the value of $I$ is close to zero, the streamwise vorticity is 
(a)

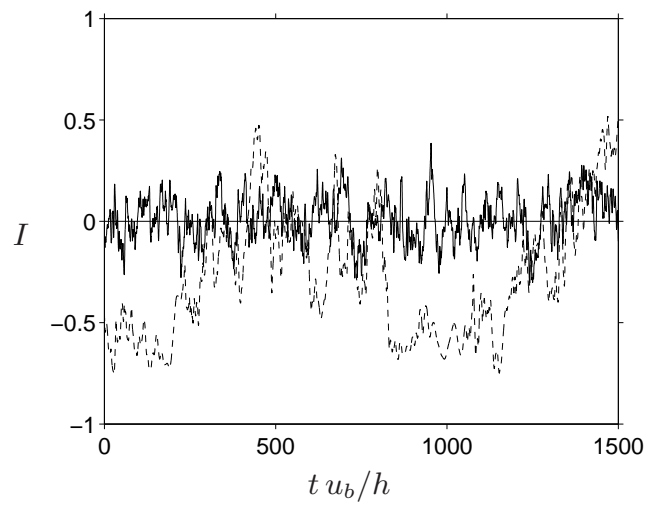

(b)

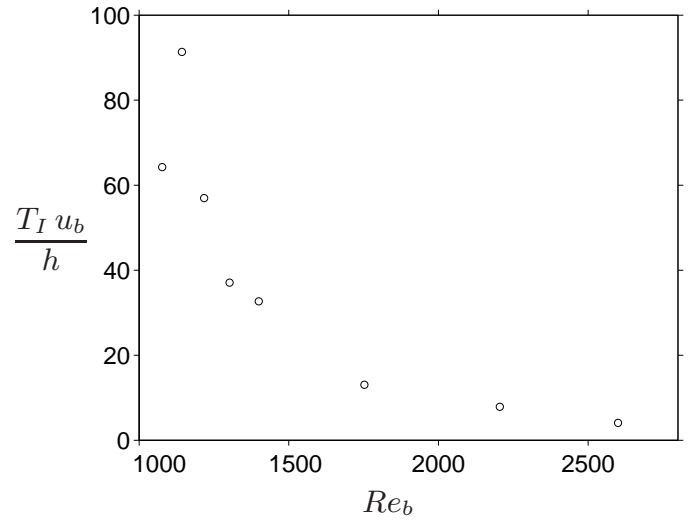

Figure 4. Temporal evolution of the indicator function. (a) $I:-, R e_{b}=2205$; ,$---- R e_{b}=1077$. In both cases $L_{x} / h=4 \pi$. (b) The average period between zero-crossings of the indicator function $I$ versus the Reynolds number for cases with long domains $\left(L_{x} / h=10.97 \ldots 12.57\right)$.

equipartitioned between the two triangular regions associated with the walls at $y= \pm 1$ and those two associated with the walls at $z= \pm 1$; this is true for an 8 -vortex state. Conversely, large absolute values of $I$ appear for the 4-vortex state, with the sign of $I$ indicating whether the vorticity $\left\langle\omega_{x}\right\rangle_{x}$ is concentrated near the walls $z= \pm 1(I<0)$ or $y= \pm 1(I>0)$. For the averaged flow patterns shown in figure $3(a, b, c)$ the indicator function takes the values $I=-0.760,0.779,-0.003$, respectively.

Figure 4(a) shows the temporal variation of the indicator $I$ for two cases with $R e_{b}=$ 1077 and $R e_{b}=2005$ (in both cases the length of the domain is $4 \pi h$ ). At the higher value for $R_{b}$ the indicator fluctuates around $I=0$ with a low amplitude and a high frequency, implying that the flow is always in the conventional 8-vortex state. For lower $R e_{b}$, however, the indicator deviates largely from $I=0$, and its sign changes after relatively long intervals of the order of $100 \mathrm{~h} / u_{b}$, as can also be seen in figure $4(b)$. This latter behavior indicates that the flow at this marginal Reynolds number exhibits the 4 -vortex state with both orientations occurring during the observation interval. If one considers a duct with infinite streamwise extension, one would observe spatial cells of finite length with a mean 4-vortex pattern. Overall, the mean over the infinite length would lead to a standard 8-vortex mean secondary flow and therefore the indicator $I$ will tend to zero for $L_{x} \gg h$.

The dependence of the amplitude of the fluctuations of $I$ upon the Reynolds number can be concluded from figure $5(a)$. Just above the critical Reynolds number, the rms value of the indicator is very high $\left(I^{\prime} \approx 0.43\right)$, then decreasing rapidly for $R e_{b} \gtrsim 1250$ and reaching a value of $I^{\prime} \approx 0.1$ for the highest Reynolds number currently investigated. The 4 -vortex state is therefore clearly a phenomenon which is only encountered at marginal Reynolds numbers. The plot in figure $5 b$ shows the amplitude of the streamwise-averaged wall shear fluctuations, which clearly exhibits a dependency upon the Reynolds number very much alike the fluctuations of the indicator function. This is a strong indication that cross-stream structures are correlated with the behaviour of the streaks (i.e. streamwise motion) which are directly responsible for the wall shear fluctuations.

\subsection{Coherent structures}

In order to determine whether there is a direct relation between coherent structures and mean secondary flow, we have performed a vortex eduction study. Coherent vortices have 
(a)

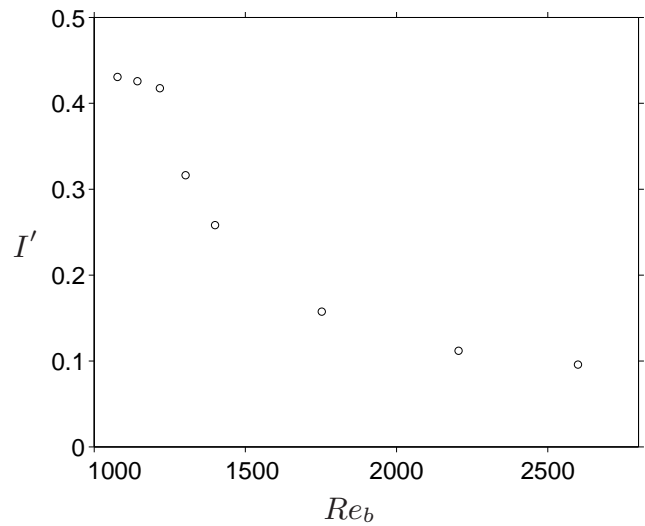

(b)

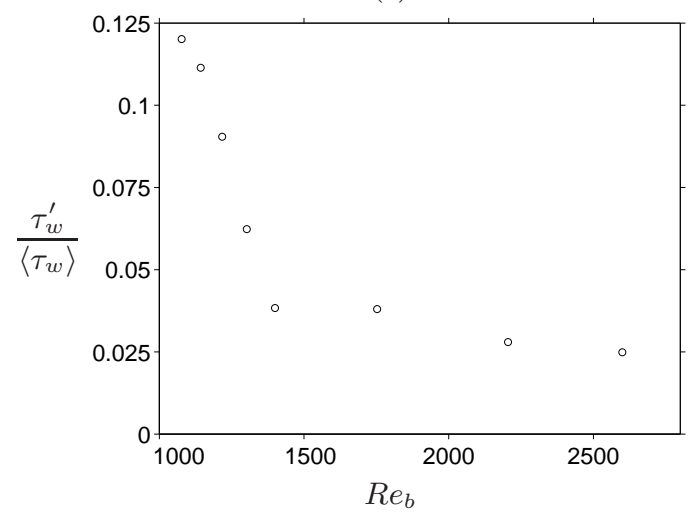

Figure 5. The rms values of $(a)$ the indicator function $I$, and $(b)$ the streamwise-averaged wall shear fluctuations, $\tau_{w}^{\prime}$, with respect to the time-averaged value $\left.\left\langle\tau_{w}\right\rangle\right)$, both as a function of the Reynolds number for cases with long domains $\left(L_{x} / h=10.97 \ldots 12.57\right)$.

(a)

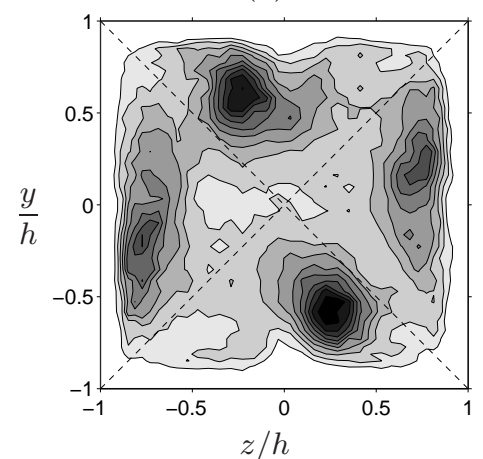

(b)

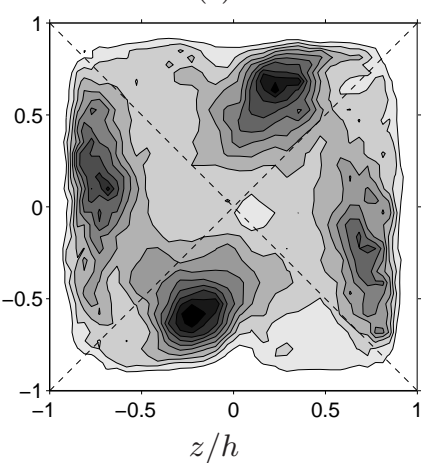

(c)

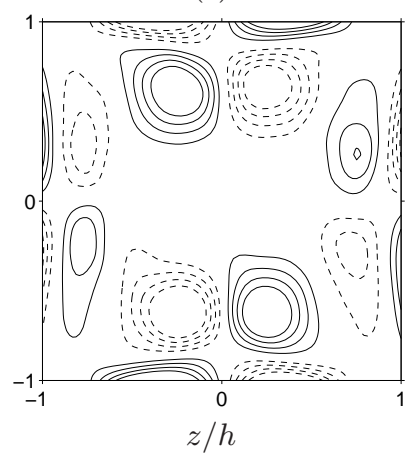

FiguRE 6. Statistical data for a case with $R e_{b}=1143$ and $L_{x} / h=4 \pi$, accumulated from 960 flow fields over a time interval of $915 h / u_{b}$. (a) Contours indicating .1(.1).9 times the maximum probability of occurrence of vortex centers with positive streamwise vorticity (shaded increasingly from white to black); (b) the probability for vortices with negative streamwise vorticity; $(c)$ the average streamwise vorticity over the same interval (isocontours with $-0.8(0.2) 0.8$ times the maximum absolute value, negative values dashed).

been detected by means of the criterion proposed by Kida \& Miura (1998): vortex cores are associated with low pressure regions and an additional condition for swirling motion without the need of introducing any threshold value. More specifically, we have applied such method to the flow in each cross-sectional plane of a number of instantaneous flow fields. In our study the locations of vortex centers are found by searching for local pressure minima in planes $(y, z)$ and checking whether these points verify the swirl condition $D<0$, where $D$ is the discriminant of the velocity gradient tensor of the flow in the cross-plane, i.e. $D=(\partial v / \partial y-\partial w / \partial z)^{2} / 4+\partial v / \partial z \cdot \partial w / \partial y$. In order to eliminate the effect of very low intensity vortices we have filtered all the contributions lower than $1 \%$ of maximum streamwise vorticity. The positions of coherent vortex centers were then stored along with the corresponding local value of the streamwise vorticity. The resulting probability of occurrence of vortex centers is shown in figure $6(a, b)$ for a Reynolds number of $R e_{b}=1143$. We observe that the local maxima of the pdf of the vortex positions bear a striking resemblance to the mean secondary flow vorticity pattern accumulated over 
the same interval (cf. figure $6 c$ ). At marginal Reynolds numbers, where the coherent structures are highly constrained by the geometry, the instantaneous streamwise vortices are practically locked into their respective positions on either side of the wall bi-sector, consistent with the signs of the secondary flow vorticity.

The same argument applies to the spanwise position of the velocity streaks. We have verified that for a marginal Reynolds number the pdf of the low-velocity streak location (identified by the local minima of the wall normal derivative of the streamwise velocity component at the wall) indeed exhibits a sharp peak around the wall bi-sector (figure omitted). This result is in turn consistent with the shape of the primary mean flow which is such that the wall shear has a local minimum at the wall bi-sector. Therefore, a lowvelocity streak is found to be statistically constrained to a narrow range of spanwise positions between two counter-rotating streamwise vortices.

\section{Concluding remarks}

Spectral direct numerical simulation of turbulent square duct flow has been performed at marginal to low Reynolds numbers considering various streamwise domain extensions. Critical values for these two parameters, allowing for self-sustaining turbulence, have been determined.

In such a marginal regime, short-time averaged velocity fields are found to exhibit a 4vortex state instead of the usual 8-vortex secondary flow pattern found at higher Reynolds numbers. It is also found that for marginal Reynolds numbers the most probable crosssectional position of the centers of coherent vortices match the mean secondary vorticity pattern. Moreover, it has been shown that the deformation of the mean streamwise velocity profile is due to the presence of a persistent low velocity streak preferentially located over the center of the edges. The matching between preferential positions of quasi-streamwise vortices and velocity streaks with the structure of the averaged flow field is a clear evidence that, for marginal Reynolds numbers, the secondary flow pattern is a direct consequence of coherent buffer layer structures.

The relationship between coherent structures and secondary flow also gives a possible explanation for the appearance of a 4 -vortex state in the regime of marginal Reynolds numbers. In this case, the dimension of the cross-section in wall units is below the one that would be needed to accommodate a complete minimal turbulent cycle on all four walls (Jiménez \& Moin 1991). Therefore, just two facing walls can be alternatively selected to give rise to a complete turbulent regeneration mechanism, while the other two faces remain in a relative quiescent state.

Two basic questions remain open. The first one concerns the validity of the proposed mechanism when increasing the Reynolds number. Indeed, at higher Reynolds numbers we should deal with the appearance of motion at different scales whereas in the marginal case the cross-stream scale of coherent structures is comparable with the duct width. The second question is related to the reason for preferential location of coherent structures. A possible heuristic explanation is based upon a simple kinematic analysis of the interaction of streamwise vorticity with a corner. Consider a generic vortex aligned with the mean flow and initially located on the diagonal close to one of the corners. Its interaction with the impermeable wall can be modeled using three image vortices and potential theory (Kawahara \& Kamada 2000). In this scenario the vortex would migrate by the induced velocity field towards a position which is consistent with the mean streamwise vorticity field observed in the real flow. In other words, there exists an automatic selection mechanism associating the position of each streamwise vortex to its actual sign of rotation. The 
quantitative importance of such mechanism and the scaling with the Reynolds number will be addressed by the authors in future communications.

The collaboration between the two groups has been supported by the Center of Excellence for Research and Education on Complex Functional Mechanical Systems (COE program of the Ministry of Education, Culture, Sport, Science, and Technology of Japan). M.U. was supported by the Spanish Ministry of Education and Science under contract DPI-2002-040550-C07-04. G.K. was partially supported by a Grant-in-Aid for Scientific Research (B) from the Japanese Society for the Promotion of Science.

\section{REFERENCES}

Bottaro, A., Soueid, H. \& Galletti, B. 2006 Formation of secondary vortices in turbulent square-duct flow. AIAA J. 44 (4), 803-811.

Brundrett, E. \& Baines, W. 1964 The production and diffusion of vorticity in duct flow. $J$. Fluid Mech. 19, 375-394.

Carlson, D., Widnall, S. \& Peeters, M. 1982 A flow-visualization study of transition in plane Poiseuille flow. J. Fluid Mech. 121, 487-505.

FAisst, H. \& Eckhardt, B. 2004 Sensitive dependence on initial conditions in transition to turbulence in pipe flow. J. Fluid Mech. 504, 343-352.

Galletti, B. \& Bottaro, A. 2004 Large-scale secondary structures in duct flow. J. Fluid Mech. 512, 85-94.

GaVRILAKIS, S. 1992 Numerical simulation of low-Reynolds-number turbulent flow through a straight square duct. J. Fluid Mech. 244, 101-129.

Gessner, F. 1973 The origin of secondary flow in turbulent flow along a corner. J. Fluid Mech. $\mathbf{5 8}, 1-25$.

Haldenwang, P., Labrosse, G., Abboudi, S. \& Deville, M. 1984 Chebyshev 3-d spectral and 2-d pseudo-spectral solvers for the Helmholtz equation. J. Comput. Phys. 55, 115-128.

Hamilton, J., Kim, J. \& Waleffe, F. 1995 Regeneration mechanisms of near-wall turbulence structures. J. Fluid Mech. 287, 317-348.

Huser, A. \& Biringen, S. 1993 Direct numerical simulation of turbulent flow in a square duct. J. Fluid Mech. 257, 65-95.

Jeong, J., Hussain, F., Schoppa, W. \& Kim, J. 1997 Coherent structures near the wall in a turbulent channel flow. J. Fluid Mech. 332, 185-214.

Jiménez, J. \& Moin, P. 1991 The minimal flow unit in near-wall turbulence. J. Fluid Mech. 225, 213-240.

Jones, O. 1976 An improvement in the calculation of turbulent friction in rectangular ducts. ASME J. Fluids Eng. 98, 173-181.

KawAharA, G. \& KAmAdA, E. 2000 Vortical structures in the corner region of a turbulent square-duct flow. Trans. JSME B 66 (643), 89-97, (in Japanese).

Kida, S. \& Miura, H. 1998 Swirl condition in low-pressure vortices. J. Phys. Soc. Japan 67 (7), 2166-2169.

Leriche, E. \& Labrosse, G. 2000 High order direct Stokes solvers with or without temporal splitting: Numerical investigations of their comparative properties. SIAM J. Sci. Comput. $22(4), 1386-1410$.

Melling, A. \& Whitelaw, J. 1976 Turbulent flow in a rectangular duct. J. Fluid Mech. 78, $289-315$.

NikuRADSE, J. 1926 Untersuchungen über die Geschwindigkeitsverteilung in turbulenten Strömungen. PhD Thesis, Göttingen. VDI Forsch. 281.

Pinelli, A., Uhlmann, M., Kawahara, G. \& Sekimoto, A. 2007 Direct numerical simulation of turbulent flow in ducts using a spectral collocation method. (in preparation).

Tatsumi, T. \& Yoshimura, T. 1990 Stability of the laminar flow in a rectangular duct. J. Fluid Mech. 212, 437-449.

Verzicco, R. \& Orlandi, P. 1996 A finite-difference scheme for three-dimensional incompressible flows in cylindrical coordinates. J. Comput. Phys. 123, 402-414. 